\title{
Pulsation dampening systems for piston pumps operating at backfilling installations
}

\author{
Tobias Lutz \& Peter Peschken \\ Putzmeister Concrete Pumps GmbH, Aichtal, Germany
}

\begin{abstract}
During metallurgical mining, most of the mined material is of no use for the industry. Only a very small portion can be used to receive the desired precious metals. The waste material is called tailings and must be transported to surface dams or mixed with additives to become a backfill paste which is transported back to the stopes. Hydraulic driven Piston pumps are one solution to deliver this high volume over long distances in a safe and economical way. However, the design principle of a hydraulically driven piston pump leads to pulsation in a delivery pipeline which is caused by the fact that the general design is based on a discontinuous working principle. The pumps are pumping one cylinder after another into the transportation pipeline. This results on one hand in flow fluctuations and consequently in pressure losses.

For optimal performance of the system it is recommended to use a pulsation dampening system with the Piston Pump. The reason is to create an approximately continuous flow of the material which is preventing pressure peaks resulting from water-based high-density solids (so-called Water Hammer Effect) with a low air content being pumped at great speed against a high pressure. Furthermore, the limited variances in flow and pressure are resulting in lower forces inside the pipeline system which leads to lower operating costs and a safer working environment for the employees on the mine site.

This paper identifies and describes the major three innovations and their different working principles of Pulsation Dampening of Paste- and Slurry Systems and discusses their application in Detail.

First System is the use of a hydraulic driven Seat valve pump with continuous Flow system. Second possibility is the use of piston pumps with hydraulic driven Pulsation Dampening System and finally, the use of Ventilated Pulsation Dampening Systems.

The job site reports show how the dampening systems are implemented at various job sites.
\end{abstract}

\section{INTRODUCTION}

\subsection{Working principle of hydraulic driven piston pumps}

Pulsation in a delivery pipeline of a reciprocating pump is caused by the fact that the general design is based on a discontinuous working principle as hydraulic-driven piston pumps are two-cylinder, single-acting, positive displacement pumps.

The pumping process can be described as follows: The Hydraulic cylinder H1 is connected to Material Piston P1 which is moving backwards and forwards in the material Cylinder M1. The same arrangement is applicable to the second hydraulic and material cylinder (Figure 1). During the pumping process, Hydraulic Cylinder $\mathrm{H} 1$ is moving backwards and sucking material into the material cylinder M1. At the same time hydraulic cylinder $\mathrm{H} 2$ is doing the counter movement and pushing the material in cylinder M2 into the delivery line. Both cylinders need 


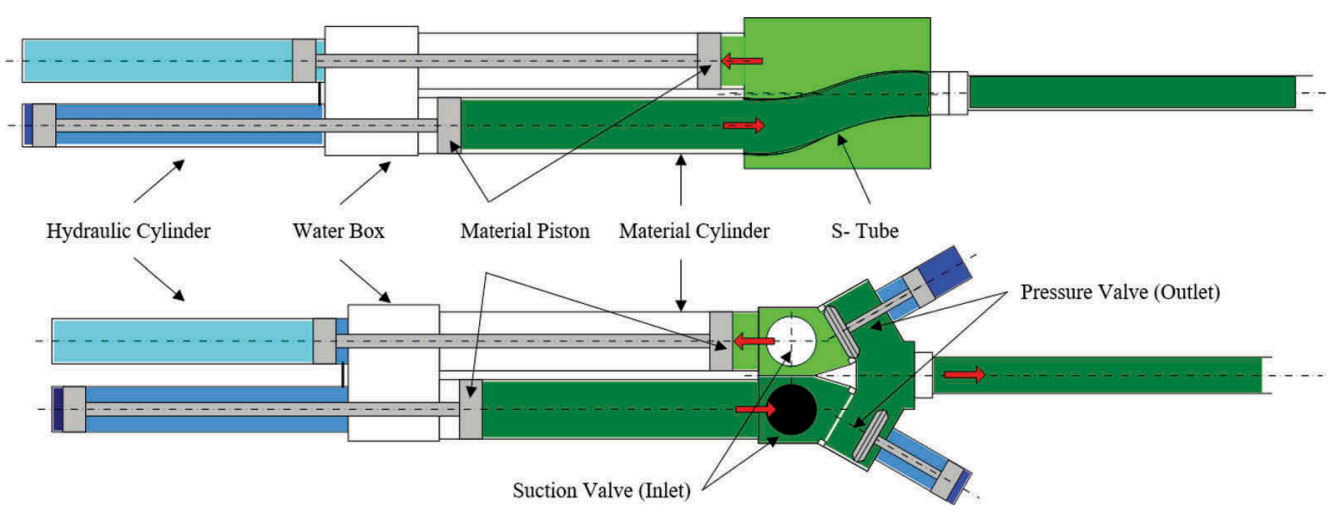

Figure 1. Schematic Layout of an S- Tube and Seat Valve Piston Pump.

to be connected to the delivery pipeline which can be either a conical shaped pipeline; which swivels in front of both cylinders (so called S- Tube); or an arrangement of hydraulically operated seat valves (see Figure 1 for illustration).

Depending on machine size and set up of the piston pump the change over of the S- Tube or the seat valves is done within $0,5-1.0 \mathrm{sec}$. Consequently, the material in the pipeline sees an immediate drop in the material flow which can be indicated in the pressure reading (Figure 2). Consequently, the static water column (along with the dissolved air) expands slightly from its compressed state. As soon as this pressure wave hits an upstream interference (valve, elbow etc.), the wave will be reflected like an "echo" and can cause a Water Hammer. During this time, the velocity of the hydraulic drive cylinders is zero.

Low volumes and/or low operating pressures of piston pumps can be operated easily with pulsations if the pipeline is designed with proper wall thickness and fixation. However, it can be recognized that there is an increasing Trend in operating volumes and operating pressure in Tailings Handlings Applications (Peschken, 2018, p. 308). This leads to the fact that Pulsation dampening is becoming an important factor for the economical evaluation of a Tailings Pipeline. Pump manufacturers for Tailings Handling Systems have to provide solutions to prevent pressure peaks, especially those resulting from water-based high-density solids (so-called Water Hammer Effect) with a low air content being pumped at great speed against a high pressure. The savings in investment are substantial if the pipeline layout can be done with only a limited pulsation therein.

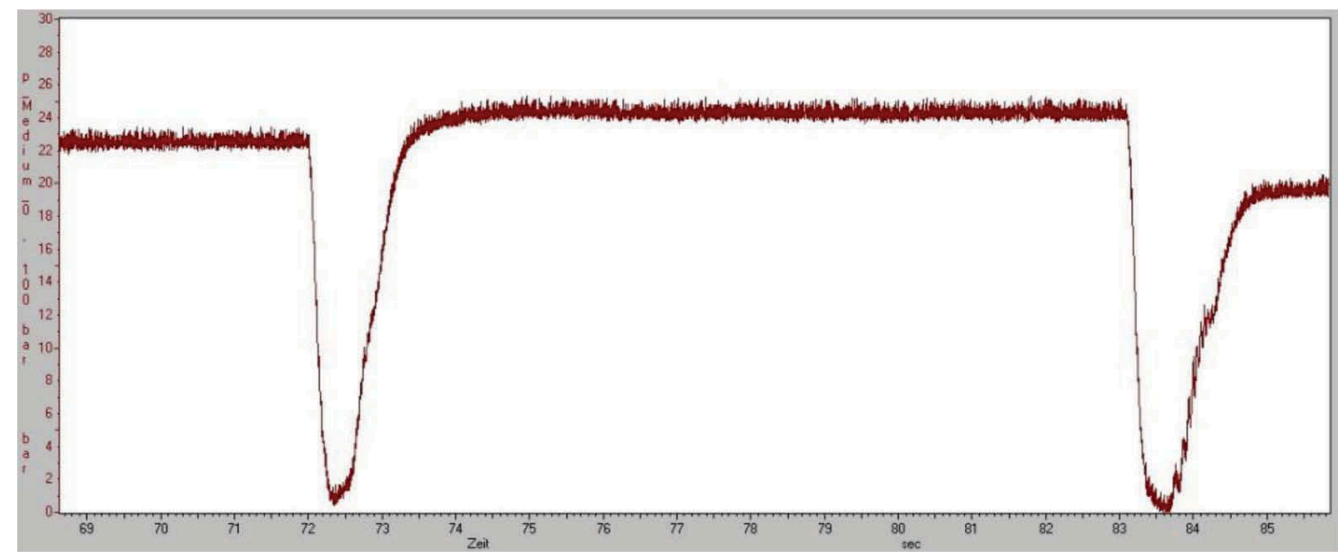

Figure 2. Typical Pressure reading of a Piston Pump. 


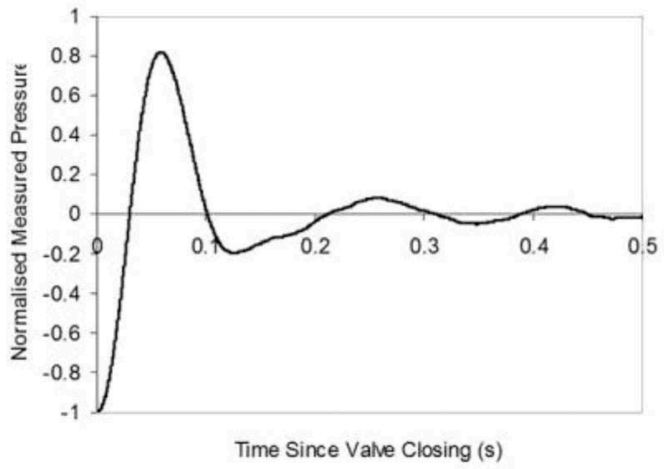

Figure 3. Normalized Pressure reading of a Water Hammer (Donebythesecondlaw, 2009).

\subsection{The water hammer effect}

The Water hammer effect is an example of conservation of energy and results from the conversion of velocity energy into pressure energy (Bregman, 2017). It is created by stopping and/or starting a liquid flow (of a Newtonian Fluid) suddenly, which creates shock waves that travel back and forth within the pumping material. A practical example of a water hammer is turning off the shower at home immediately. "The hammer occurs because an entire train of water is being stopped so fast that the end of the train hits up against the front end and sends shock waves through the pipe. This is like a real train, instead of slowing to a stop, it hits into a mountain side. The back of the train continues forward even though the front cannot go anywhere. Since the water flow is restricted inside the pipe, a shock wave of incompressible water travels back down the pipe." (OMEGA Engineering, 2018).

This shock wave can be indicated as a variation in pressure (Dp). It is creating a pressure wave almost like sound waves, traveling upstream the pipeline has "all of its kinetic energy of motion and that due to compression is converted into pressure energy" (Evans, 2011).

"The first person to describe this effect was the Russian scientist Joukowski. He showed both theoretically and experimentally that there is a maximum pressure that can be produced, known now as the "Joukowski head" or "Joukowski pressure" depending on the units of its expression (Ord, S.C.; Stopford Projects Ltd, UK, 2006).

It is given by the following formula

$$
h=\frac{v * c}{g}
$$

where

$\mathrm{c}=$ speed of the sound wave in the pipe, known as the "wave celeric", $\left[\mathrm{m} / \mathrm{s}^{1}\right]$

$\mathrm{v}=$ initial velocity of the liquid, $[\mathrm{m} / \mathrm{s}]$

$\mathrm{g}=9.81\left[\mathrm{~m} / \mathrm{s}^{2}\right]$

$\mathrm{h}=$ Joukowski head, $[\mathrm{m}]$

Thus, for water in a rigid pipe, where the speed of the sound wave in the pipe is (for example) $1500 \mathrm{~m} / \mathrm{s}$, for an initial water velocity of $2,5 \mathrm{~m} / \mathrm{s}$, then suddenly stopping the flow with a rapid valve action will result in a head of about $380 \mathrm{~m}$, or 38 bar. The maximum closure time that will give this head is given by

1. in water it is $1.484 \mathrm{~m} / \mathrm{s}$. However, for Slurry or even paste the value is unknown and project specific 


$$
t=\frac{2 * L}{c}
$$

where

$\mathrm{L}=$ length of pipe in $[\mathrm{m}]$

$\mathrm{t}=$ time, which is the time it takes for a pressure wave to travel the pipe length and back again [sec].

Hence if the pipe is $2.500 \mathrm{~m}$ long, the maximum closure time is about $3.33 \mathrm{~s}$, ie a closure in this time or less produces a Joukowski head. "For closure times up to 10 times this, [ie $33.3 \mathrm{~s}$ ], the pressure is reduced in about inverse proportion which would result" (Ord, S.C.; Stopford Projects Ltd, UK, 2006, p. 4) in a pressure increase of 3,8 bar for the previous example.

Due to the pressure discontinuity, the passing of the wave front produces a force to the pipeline and its support at the next bending of the pipe.

Thus, for a $150 \mathrm{~mm}$ diameter pipe, the passage of the 38-bar wave described above produces a force of

$$
F=\frac{\pi}{4} * D^{2} * P
$$

where

$\mathrm{F}=$ force, $[\mathrm{N}]$

$\mathrm{P}=$ the pressure equivalent of the Joukowski head $[\mathrm{Pa}]$

In our example, the Pipeline would see a Force of $67 \mathrm{kN}$, which is a significant force and could lead to damage of the pipeline, support or even the pumping system. "Sadly, that's not the full story. The forces and pressures calculated above are for specific, fast events with water as medium and therefore for ideal Newtonian Fluids. There are lots of shades of grey between a Joukowski event and a gradual change in fluid speed that gives no perceptible surge forces. So, in many cases, a more complex analysis or even tests have to be done to ascertain if a pipe is at risk" (Ord, S.C.; Stopford Projects Ltd, UK, 2006, pp. 2 - 5). For different material characteristics such as different rheological behaviors (thixotropic pastes; Slurries; cake pastes etc.) it is even more difficult to predict whether the risk of a Water Hammer exists or not. Nevertheless, it can be stated that the more and the bigger the solids (by volume) are within the pumping media the lower the risk of a water hammer as the speed of the sound wave in the pipe will be lower.

\subsection{Consequences of pulsations in the pipeline}

Pulsation in the pipeline is an unwanted circumstance of every designer and every operator of Slurry- and Paste Systems. The reasons are at hand. As described in the previous chapter, the pulsation may cause mechanical vibrations and fatigue failures which can lead to serious damage at the pipeline and its related components such as foundations and supports due to the high level of fluctuating forces. The lifetime of valves, flanges and other apertures is limited as they are affected by the pulsations as well. If the pulsations are considered during the design phase, the entire pipeline becomes an even higher capital investment of the operator. Consequently, it is a calculation between adding a pulsation dampening system to the pump or additional supports to the pipeline. In addition to that, the pulsations in the pipeline are leading to higher noise emissions to the environment of the pipeline which can cause issues for surface Pipelines (from Tailings Ponds and Ash ponds). 


\section{PULSATION DAMPENING SYSTEMS FOR HYDRAULICALLY DRIVEN PISTON PUMPS}

\subsection{Nitrogen charged Diaphragm Dampener}

The first use of pulsation dampening systems was done with Nitrogen charged Diaphragm Dampeners as shown in Figure 4. Nitrogen charged Diaphragm Dampeners are common practise in hydraulic fluid systems or other fluid pumping systems like metering systems. The function of this pulsation dampener is based on the compression of a nitrogen gas Diaphragm which is usually designed in butyl- or fluoride rubber (Lutz-Jesco GmbH, 2018).

During the discharge of the piston pump the pumping material is squeezed into the Dampener and is compressing the Nitrogen Diaphragm. During the changeover of the S- Tube or seat valve, the material is pushed into the pipeline by the Nitrogen pressure in the Pulsation Dampening Diaphragm. As the volume of this bladder type Pulsation Dampeners is only limited, the quantity of installed dampeners must be increased for high flow rates. Consequently, big Tailing installations can be equipped with $>10$ of these Diaphragm Dampeners.

During the operation of various Slurry Systems with Piston pumps, there have been several issues reported (Hövemeyer \& Zey, Industrial Pump Technology - Treatment, pipe transport and storage of high-density substances, 2019). The main concern is that the entire Diaphragm is sucked into the pipeline due to the high velocity and rapid change over of a piston pump. Furthermore, the system is very sensitive towards foreign bodies as stones or other spiky particles can harm the Diaphragm which lead to excessive maintenance work at the dampeners. For this reason, this technology is not used for new designed installations and plants. The industry improved the dampening methods towards three different working principles. First, the modification of the Piston pump which leads to an almost Pulsation Free operation. Secondly to a pulsation dampening system which includes an additional delivery cylinder and finally a ventilated dampening system which is a modification of the Nitrogen charged Diaphragm Dampener.

\subsection{Constant Flow}

The Constant Flow System is the best in class pulsation dampening system for seat valve - or ball valve pumps as it is a "no pulsation" dampening system. It is a pulsation avoidance system because it changes the way of operation of a piston pump, so the pulsation is not even created. Consequently, no additional mechanical components must be installed within the delivery pipeline. The only required Equipment is a Seat Valve or Ball Valve Piston Pump equipped with a Constant Flow Hydraulic Power Pack. With this system the material flow is kept at an almost even level which consequently reduces the pressure peaks significantly. This continuous material flow is achieved as the connection between the hydraulic cylinders is eliminated. Every cylinder of the piston pump is connected to an independent Hydraulic Oil Pump and acting as a single, independent piston pump. This enables the machine to perform the suction stroke faster than the pressure stroke to pre-compress the material in the material cylinder

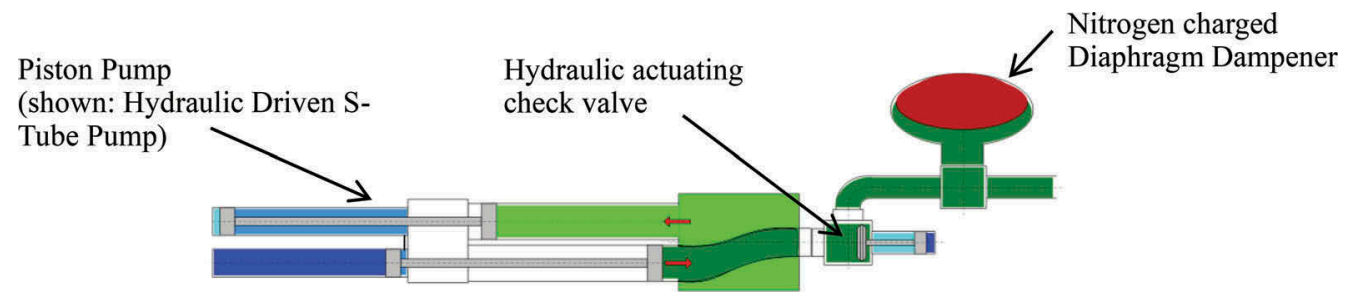

Figure 4. Schematic Layout of a Piston Pump with Nitrogen charged Diaphragm Dampener. 


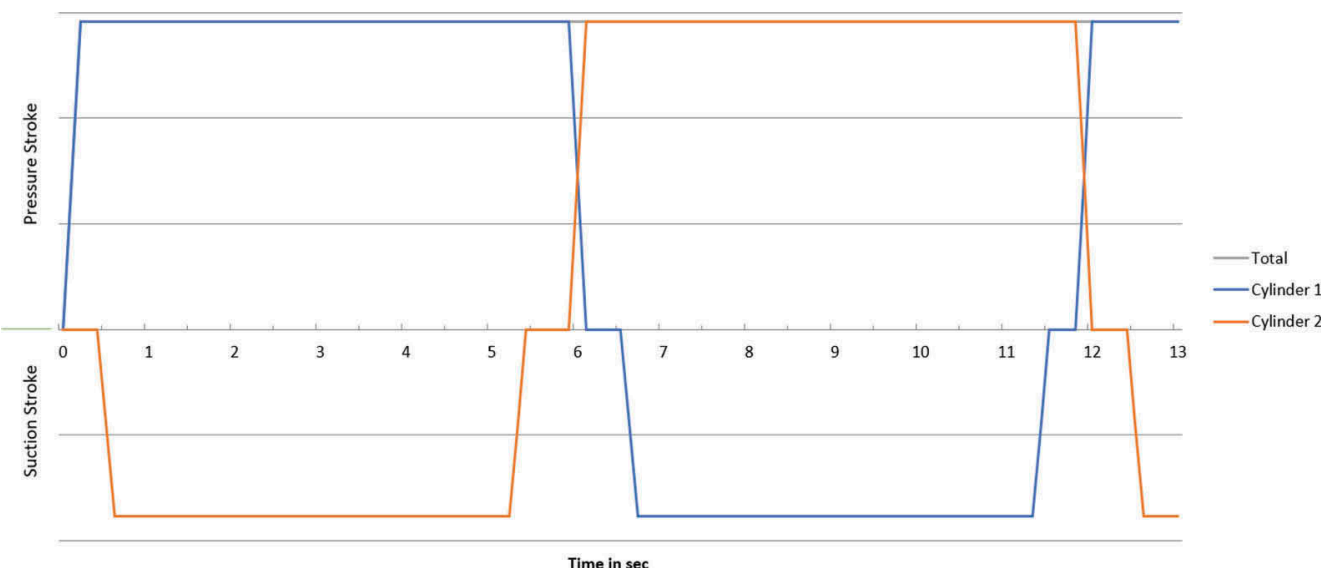

Figure 5. Constant Flow system - Working Concept.

before the pressure valve is opened. The Constant Flow System working principle is shown in Figure 5.

Cylinder 1 performs its pumping stroke, cylinder 2 starts compressing the material in the material cylinder. As piston 1 nearly reaches its end position, the speed will be reduced and piston 2 softly takes over the load. At this moment the speed of both cylinders is equal. After reaching the end position, piston 1 moves quickly back and sucks paste into the delivery cylinder while piston 2 pushes paste into the pipeline and performing its pumping stroke. The related pressure valve will close and now cylinder 1 does its pre-compression while cylinder 2 is finishing its cycle (Hövemeyer \& Zey, 2019).

This Control Philosophy leads to an almost even Flow and Pressure Curve of the pump, directly after the pressure outlet as shown in Figure 6.

Besides the Pulsation Elimination, the Constant Flow system has additional benefits for the operator. The system doesn't need any additional mechanical or electrical components in the pipeline or in the pumping material. Consequently, no additional components must be maintained and operated. In addition to that, the system can be used for various, different materials with different flow rates up to a pipeline pressure of 150 bar without adjustment thereof. It doesn't matter whether the paste or slurry contains cement and tends to harden as the

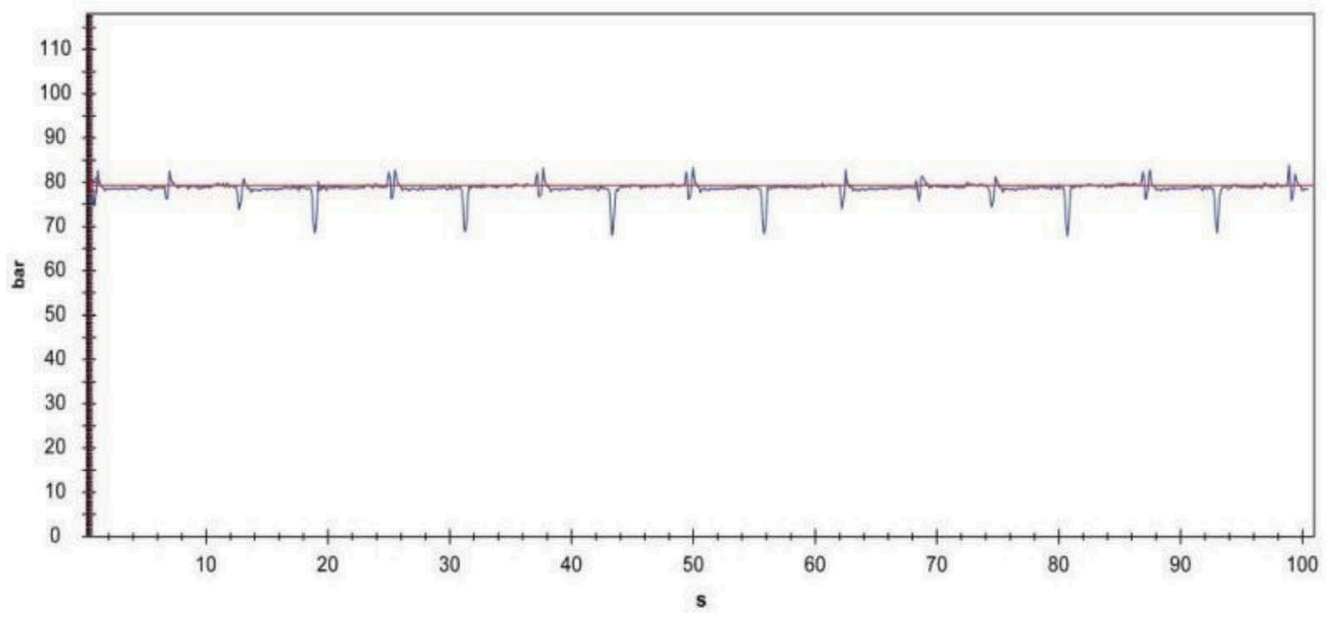

Figure 6. Constant Flow Pressure reading. 
Constant Flow system can be cleaned out completely. This has been proven by operators in multiple plants worldwide since the invention of the Constant Flow system. Due to the continuous flow of the material, the paste or slurry doesn't need to be accelerated with every stroke from 0 to full speed. This results in a lower pressure level in the pipeline compared to the operation with Piston Pumps without Constant Flow system. Furthermore, the energy consumption of the electric motor(s) is homogenized as the load is not dropping during the changeover as it is with Pump Systems without Constant Flow. This relieves the variations in the power grid and improves its reliability. Finally, the piston velocity during the pumping stroke is slower as compared to machines without Constant Flow control (due to the changeover). This results in a lower wear rate and higher lifetime of the entire system.

\subsection{Hydraulic Pulsation Dampening System (HPD)}

The Hydraulic Pulsation Dampening System is the first real pulsation dampening system for any kind of Piston Pump application and is used when slurry or paste contains sand or coarse material. The Hydraulic Pulsation Dampening System literally acts as a third delivery cylinder, which is connected to the delivery pipeline by a T-flange immediately behind the pump outlet flange. It is dampening the pulsation of the system as it delivers material into the pipeline during the changeover of the S- Tube or the valves. It is either connected to the Hydraulic Power pack of the Piston Pump or is equipped with an independent one.

Tailings with a maximum grain size of $<25 \mathrm{~mm}$ can be pumped with Seat Valve pumps or S- Tube Pumps. As soon as the grain size exceeds $25 \mathrm{~mm}$, the material should be pumped only with S- Tube piston pumps. If the Hydraulic Pulsation Dampening System is used with a Hydraulic Driven S- Tube Piston Pump, the pump has to be equipped with a hydraulic actuating check valve directly before the Hydraulic Pulsation Dampening System to prevent any backflow of the material into the wrong direction of the pipeline (cp. Figure 7). The Hydraulic Pulsation Dampening System is charged during each delivery stroke of the pump. The content of this third cylinder is actively pushed into the delivery pipeline during the changeover of the Piston pump. This closes the delivery gaps created by the change-over process and creates an almost even flow of material and consequently a much smoother pressure reading within the pipeline.

The Hydraulic Pulsation Dampening System is designed as a stand-alone unit and driven by the common power pack of the piston pump or driven by a dedicated, independent power pack. Hence it is very interesting for customers with existing paste plants as it can be used for new installations or as a retrofit for existing pump lines. The working principle of a Piston pump with the Hydraulic Pulsation Dampening System Pulsation dampening system can be described as follows. One material cylinder of the piston pump performs its pumping stroke.

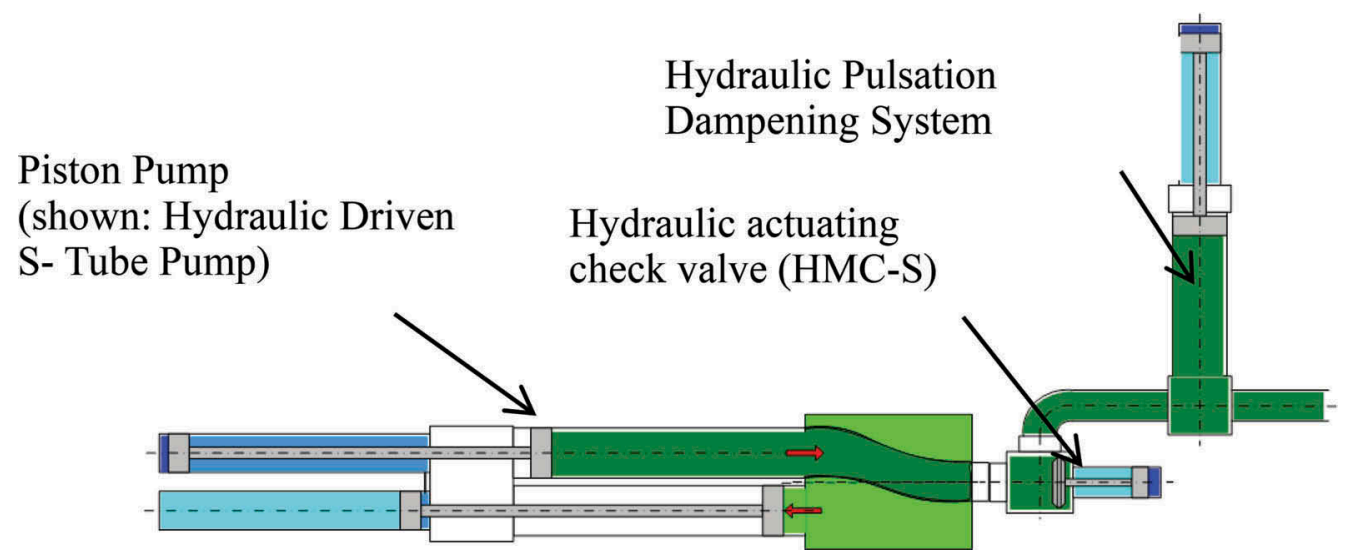

Figure 7. Schematic Layout of a Piston Pump with Hydraulic Pulsation Dampening System. 

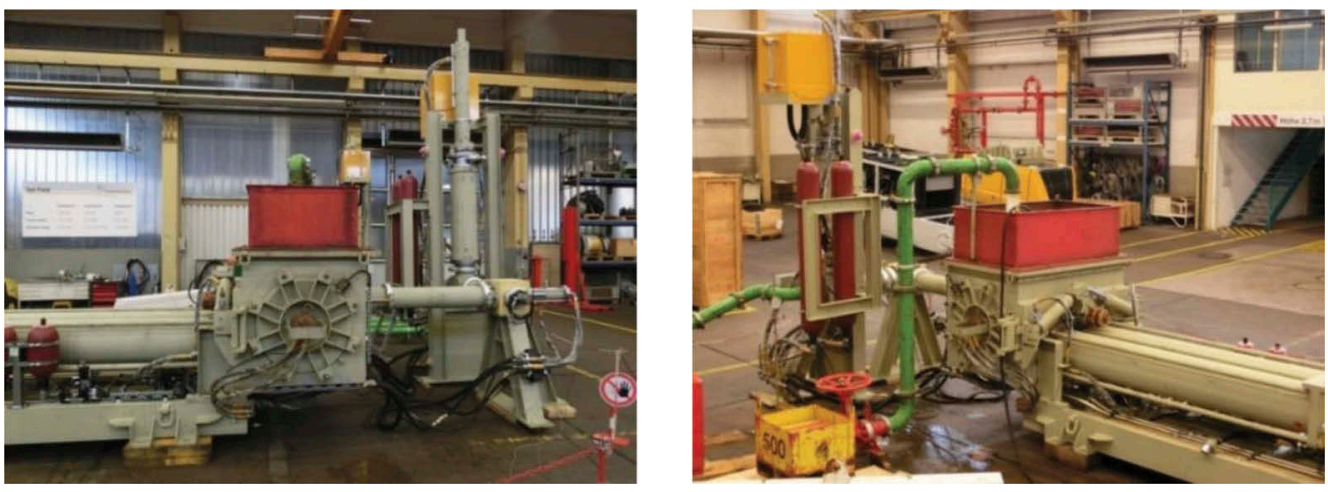

Figure 8. Hydraulic Driven S- Tube Pump with Hydraulic Pulsation Dampening System.

After reaching the end position, the Hydraulic actuated check valve is closing, and the Hydraulic Pulsation Dampening cylinder is extracting (and consequently pushing material into the delivery pipeline). As soon as the shift over of the seat valve or the shift over of the STube is finished, the Hydraulic actuated check valve is opened, and the material cylinder of the piston pump is delivering material in the pipeline. At the same time the Hydraulic Pulsation Dampening System stops extracting material into the pipeline and reversing its direction, so it is recharged for the next change over. The pressure result can be seen in Figure 9:

Hydraulic Pulsation Dampening System Pulsation dampening systems can achieve a dampening rate of approx. $60-70 \%$. The dampening rate is calculated by the ratio between the nominal pressure and the pressure drop during the shift over as indicated and described in Figure 9. This shows that the pulsation is not completely eliminated but water hammers are not possible anymore. Consequently, the design forces for the pipeline and its supports are tremendously lower. The advantage of this System is that it is the best and most reliable pressure dampening solution for hardening material (like cemented paste or slurry) with coarse material

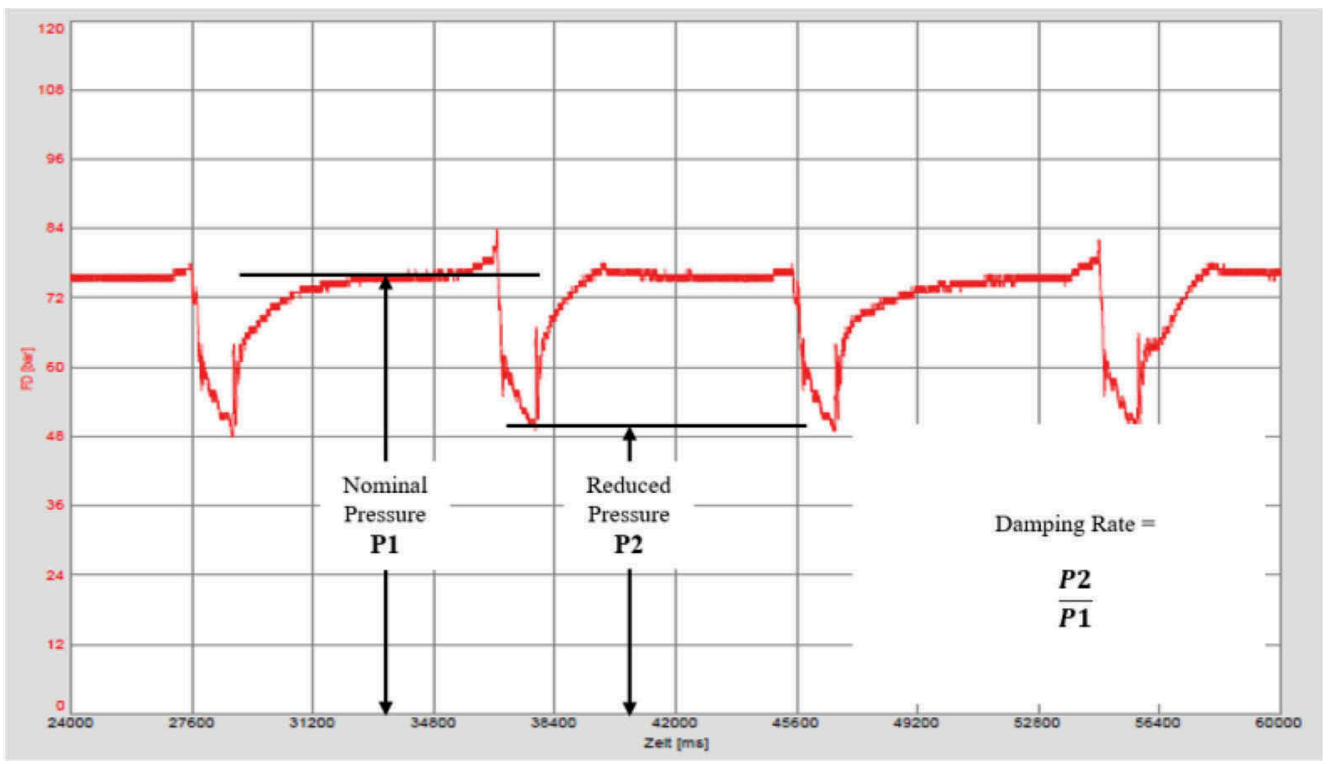

Figure 9. Pressure Reading of a Piston Pump with Hydraulic Pulsation Dampening System. 
(grain size: above $25 \mathrm{~mm}$ ). As it is designed for concrete material, the combination of S- Tube piston pumps with Hydraulic Pulsation Dampening System is insensitive towards foreign bodies like stones. Consequently, it is the proven choice of operation for Backfilling plants with coarse concrete and Ash handling systems where Bottom- and Fly- Ash are pumped as a mixture to the stope or Ash pond. As it can be operated with an independent power pack the Hydraulic Pulsation Dampening System can be easily retrofitted to any Piston Pump System.

\subsection{Ventilated pulsation dampening system}

The Ventilated Pulsation Dampener has been developed to reduce pressure drops in the pipeline with a maximum efficiency and a minimum required additional energy (Freitag, 2015). Like the Hydraulic Pulsation Dampening System, the Ventilated Pulsation Dampener literally acts as third (or multiple) delivery cylinder(s), which is connected to the delivery pipeline by a T-flange immediately behind the pump outlet flange. It is dampening the pulsation of the system using compressed air which is acting as a spring.

The Ventilated Pulsation Dampener consists of the actual dampening unit (which consists of multiple dampeners), an air accumulator and distribution unit, a high-pressure compressor and the patented intelligent control Unit (see Figure 10).

Important note: Due to the design of the Ventilated Pulsation Dampener it can be only used for non-hardening slurries and paste. Cemented paste must not be pumped through this system. Like the previously described Hydraulic Pulsation Dampening System, the Ventilated Pulsation Dampener is designed as a stand-alone unit. Therefore, it can be used for new installations or as a retrofit for existing pump lines as shown in Figure 11.

During the pump stroke of the pump, the pre-compressed air in the dampeners gets further compressed by the medium through which the medium rises in the dampeners. During the changeover of the seat valves or the S-tube, the compressed air presses the medium downwards into the conveying pipe, whereby the pressure collapse is reduced. The amount of air needed is detected by a pressure sensor in the dampening unit, calculated by the patented control system, generated from the compressor and provided from the accumulator and distribution unit (Freitag, 2015). As soon as the system is operating at a constant level, the compressor is automatically turned into stand-by mode and no additional energy is consumed anymore. Small changes in variations are compensated by the accumulator and distribution unit. As soon as the flow/pressure increases significantly, the compressor is turned on again and recharging the accumulator and distribution unit.

In regards of the Dampening rate we can rely on the physical law where more dampening volume will achieve higher dampening rates and consequently lower pressure drops during the changeover phase of the piston pump. From an economical point of

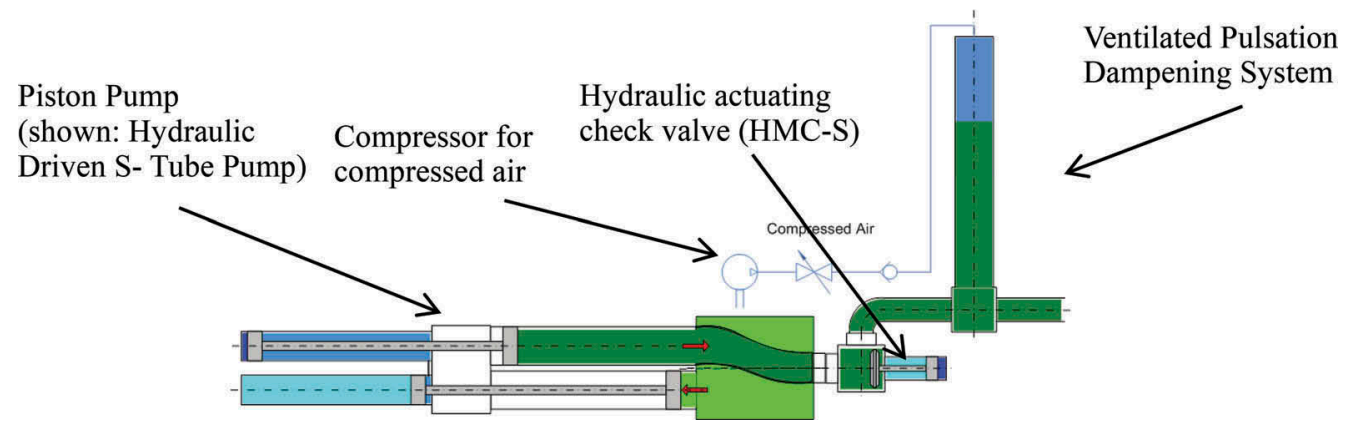

Figure 10. Schematic Layout of a Piston Pump with Ventilated Pulsation Dampening System. 


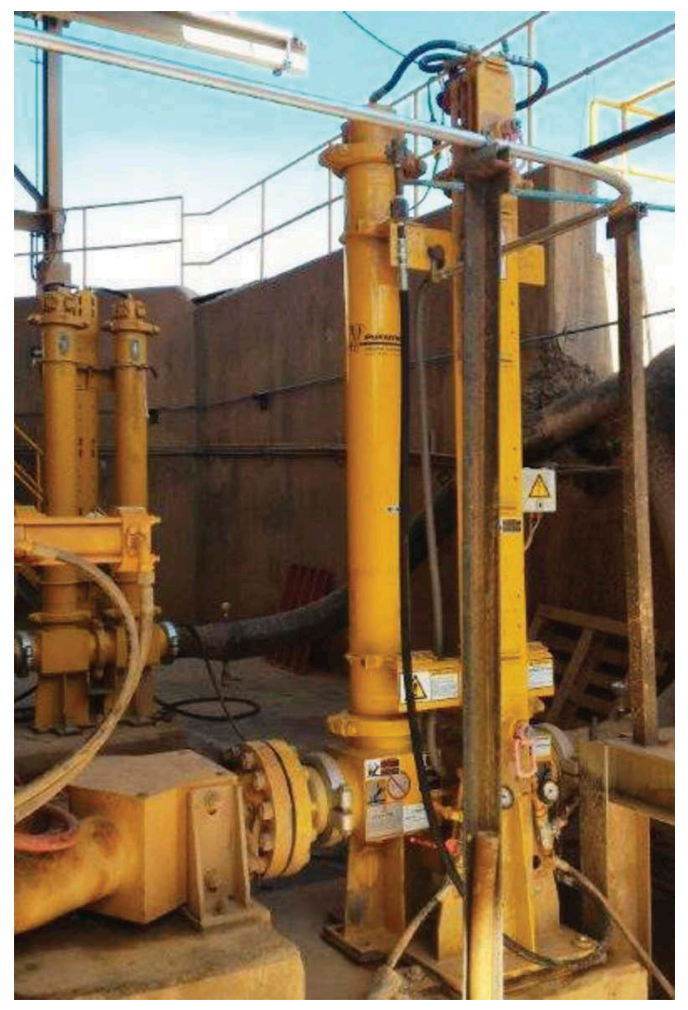

Figure 11. Installed Ventilated Pulsation Dampening System for Tailings Handling.

view, it can be outlined that the layout of the system should be done with a dampening rate of approx. 70\% (see Figure 12).

The main advantage of the Ventilated Pipe Dampener is the low consumption of energy combined with no additional wear parts in the paste or tailings flow as it is using compressed air as a spring that stores energy during continuous operation (the compressor is only needed if the output or the pressure are changing). With an intelligent Control System, the Ventilated Pulsation Dampener can be automatically operated in different pressure levels without any manual adjustment. Due to the proven technology, the system is reliably eliminating water hammer in the pipeline while it is also easy to clean and maintain as no wear parts (like membranes) are necessary. The Ventilated Pulsation Dampener is a perfect Pulsation Dampening System for nonhardening pumping materials and applicable for new installations as well as an easy retrofit for all kinds of existing pumps.

\section{PROJECTS WITH PULSATION DAMPENING SYSTEMS}

Bulyanhulu Gold Mine: At Bulyanhulu up to $80 \mathrm{~m}^{3} / \mathrm{h} 10$ inch slump paste were pumped over $2500 \mathrm{~m}$ with a maximum delivery pressure of 80 bar. The seat valve pump pushes the paste through a DN 200 pipeline. Pressure dampening was achieved by a HPD driven by the power pack of the main pump.

Eti Bakir Copper Mine: At Eti Bakir up to $65 \mathrm{~m}^{3} / \mathrm{h}$ cemented paste is pumped $830 \mathrm{~m}$ horizontal, then $290 \mathrm{~m}$ down and then over different distances underground to various chambers. The maximum delivery pressure is 150 bar. The seat valve pump of 


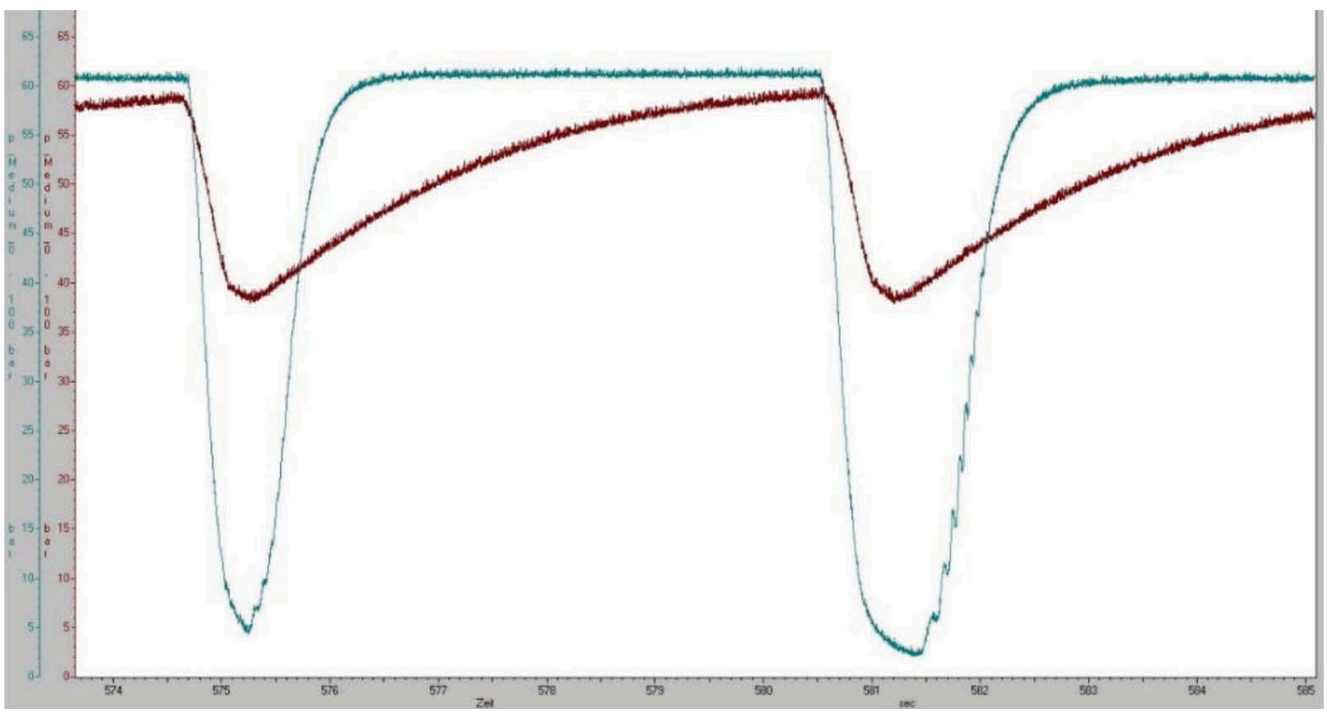

Figure 12. Pressure reading of a Piston Pump with and without Ventilated Pulsation Dampener.

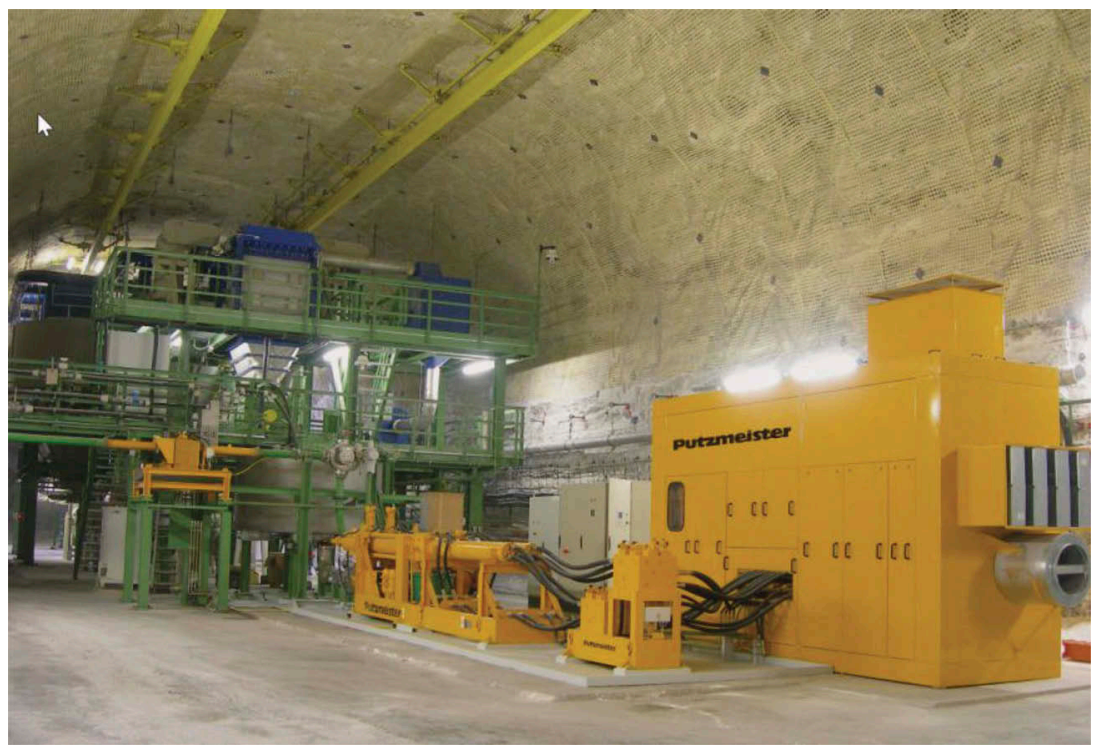

Figure 13. Underground Backfill Plant at Kali \& Salz.

type HSP 2180 HPS, driven by a Hydraulic Power Pack with $315 \mathrm{~kW}$ motor, is equipped with the PCF pressure dampening system. The paste process is typical for a gold or copper mine. The fine grained tailings with a maximum grain size of $110 \mu \mathrm{m}$ is put into a thickener; the thickener underflow is pumped into a surge tank and the slurry from the surge tank is again thickened by disc filters. The cake from the disc filters is mixed with cement and slurry and then pumped underground with the piston pump into the chambers for stabilizing the mine. 


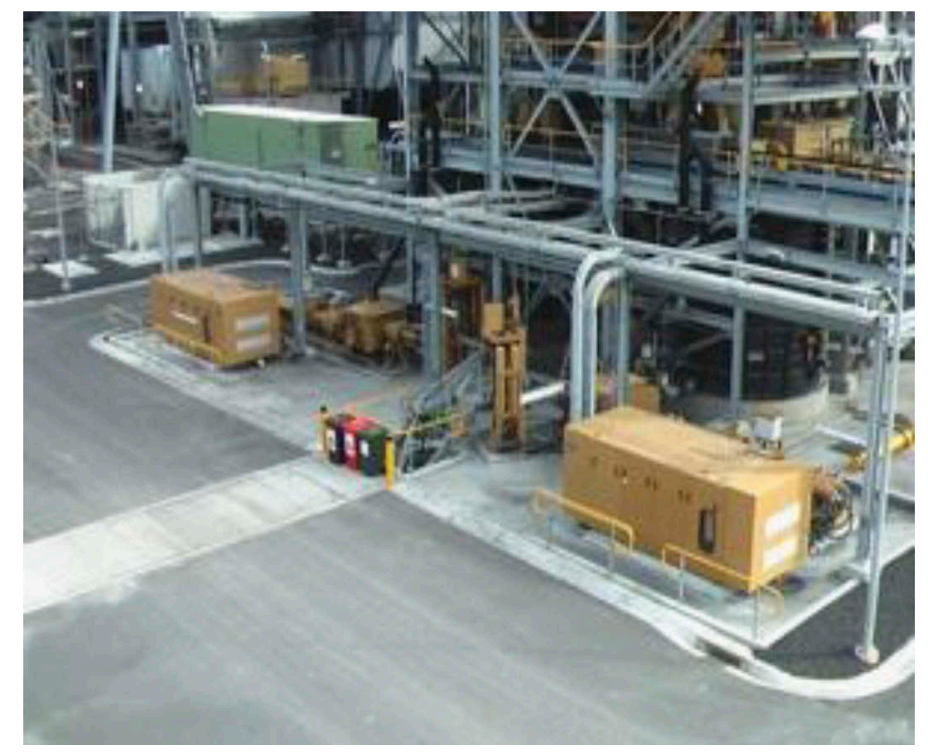

Figure 14. Fly- and bottomash pumping station at Kogan Creek.

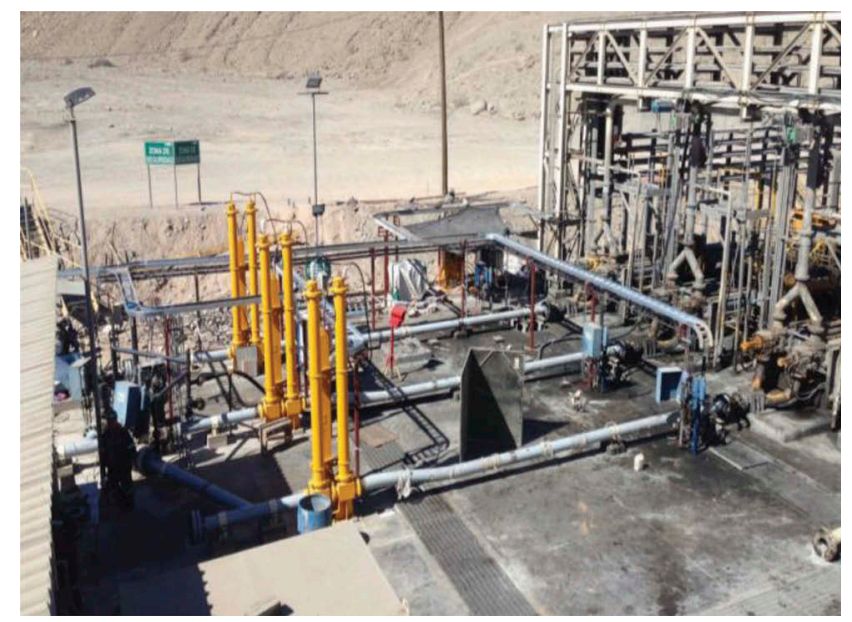

Figure 15. Tailings pumping plant at Coemin with VPD.

Kali \& Salz: Seat valve pump and power pack with PCF system are placed underground. $\mathrm{Up}$ to $80 \mathrm{~m}^{3} / \mathrm{h}$ fine grained potash with brine and binder is pumped underground for stabilisation. The parts in contact with this aggressive slurry are made of stainless steel.

Kogan Creek: At Kogan Creek flyash with up to $50 \mathrm{~mm}$ bottom ash particles are pumped over 5.500 meters back into the open pit mine. The KOS 25.100 HP pump, driven by a Power pack of type HA $250+250$ E conveys up to $152 \mathrm{~m}^{3} / \mathrm{h}$ at a max. delivery pressure of 69 bar. Pressure dampening is done by a HPD $280 / 750$ driven by the main power pack. The pipeline diameter is $150 \mathrm{~mm}$ to achieve the necessary speed to avoid sedimentation. One pump system is working, one standby.

Coemin: At the Coemin Copper Mine up to $162 \mathrm{~m}^{3} / \mathrm{h}$ fine grained tailings are pumped at a maximum delivery pressure of 94 bar. Three HSP 25.100 HPS, each one driven by a HA 
$630 \mathrm{E}$, are installed. Pressure peak dampening of each pump is done by a Ventilated Pipe Dampener with 2 pipes each.

\section{CONCLUSION}

Pulsation Dampening is an essential element for Tailings handling and Paste Backfilling applications and will become even more important for future projects. Due to increasing delivery volumes, -distances and pressures it is going to be crucial to install the right dampening solution to secure a reliable and safe working condition of the piston pump system. Furthermore, pulsation dampening systems are helping to reduce the overall installation costs as the wall thickness of the delivery pipeline and its safety factors could be optimized accordingly. This paper outlined three different pulsation dampening solutions to avoid or to reduce the shocks in the pipeline significantly.

The Constant Flow System, which is obviously no dampening system as it avoids the pressure pulses in the pipeline due to its independent working cylinders. This system is the preferred solution for all new designed cemented Paste- and Slurry Systems and can be operated until 150bar continuous delivery pressure. A ventilated pulsation dampening system Ventilated Pulsation Dampener is the economically friendly solution for tailings handling if the material is not hardening. The additional benefit is that this system can be retrofitted to any existing Piston pump station. Due to the patented intelligent control, the ventilated pulsation dampening system is only consuming a minimum of energy during continuous operation. Finally, the paper presented the hydraulic pulsation dampening System Hydraulic Pulsation Dampening System which is the only solution for Backfilling systems with coarse material that are operated with S- Tube Pumps. It can be supplied for new project but as well be retrofitted to existing pumping stations.

\section{REFERENCES}

Bregman, A. (2017, May 9). Water Hammer. Retrieved July 15, 2019, from valvemagazine.com: https:// www.valvemagazine.com/magazine/sections/back-to-basics/8418-water-hammer.html

Donebythesecondlaw. (2009). Wikipedia. Retrieved February 27, 2018, from Water hammer pressure pulse: https://en.wikipedia.org/wiki/Water_hammer\#/media/File:Water_hammer_pressure.jpg

Evans, J. (2011). Pumps \& Systems. Retrieved February 27, 2018, from The Causes of Water Hammer (Part One): https://www.pumpsandsystems.com/topics/pumps/pumps/causes-water-hammer-part-one

Freitag, U. (2015, November 25). TI 151124 - Ventilated Pulsation Damper (VPD).

Hövemeyer, D., \& Dr.-Ing. Richter, A. (2014, April 11). Challenges of pump and process engineering for the transport of solids with hydraulic piston pumps. (B. Ernst \& SohnVerlag für Architektur und technische Wissenschaften GmbH \& Co. KG, Ed.) Mining Report (150 No 1/2). doi:https://doi.org/ $10.1002 /$ mire. 201400003

Hövemeyer, D., \& Zey, W. (2019). Industrial Pump Technology - Treatment, pipe transport and storage of high-density substances. (Karl Schlecht Stiftung (KSG), Ed.) Aichtal.

Lutz-Jesco GmbH. (2018, November 5). Pulsation Damper PDM. Retrieved from http://www.lutz-jescoa sia.com/LJ-Pulsation_Dampener_PDM-EN-MB.pdf\#targetText $=\mathrm{A} \% 20 \mathrm{basic} \% 20$ distinction $\% 20 \mathrm{is} \%$ 20made, is $\% 20$ compressed $\% 20$ to $\% 20$ damping $\% 20$ volume.

OMEGA Engineering. (2018). OMEGA Engineering. Retrieved February 27, 2018, from https://www. omega.co.uk/techref/waterhammer.html

Ord, S.C.; Stopford Projects Ltd, UK. (2006). Water Hammer - Do we need to protect against it? How to predict it and prevent it damaging Pipelines and Equipment. IChemE(SYMPOSIUM SERIES NO. 151), $1-20$.

Peschken, P. (2018). Hydraulic Driven Piston Pumps for the Transport of Pastes and Slurries in the Mining Industry. Mining Report Glückauf(No. 4), pp. 306-317. Retrieved July 22, 2019, from https:// mining-report.de/wp-content/uploads/2018/08/MRG_1804_KOSK_Peschken_180811.pdf

Putzmeister. (2014). Putzmeister Industrial Technology in Mining. Retrieved April 25, 2019, from Putzmeister: https://www.putzmeister.com

The Process Piping. (2017, September 4). Retrieved from Water Hammer in Process Plant: https://www. theprocesspiping.com/water-hammer/ 